\title{
Analysis of The Use of Phase Change For The Safe Operation of Electrical Equipment
}

\author{
Zuzana Kolková ${ }^{1}$, Peter Hrabovský, ${ }^{2}$ Jozef Matušov ${ }^{3}$, Gabriela Belavá ${ }^{4}$, Andrea Dávidiková ${ }^{5}$ \\ ${ }^{1}$ Research centre, University of Žilina, Univerzitná 8215/1, 01001 Žilina, Slovakia
}

\begin{abstract}
Cooling is currently a serious issue for any manufacturing company, no matter what it is dealing with. Cooling affects all areas of production and relates directly to primary production or to the completion of final products. The ability to accurately manage large-scale electrical voltages has enabled many effective and significant innovations. These devices require high-efficiency electronics cooling. Dielectric fluids are also safe in contact with electronics. They can be used to cool various devices. They work without compromising the health of workers or harming the environment. Special devices called heat pipes are used more and more widely to transfer the excessive heat to a colder environment. The mechanical moving parts are removed. Pulsating (or oscillating) heat pipe present promising alternatives for the removal of high localized heat fluxes to provide a necessary level of temperature uniformity across the components that need to be cooled. PHP is a capillary tube (with no wick structure) bent into many turns and partially filled with a working fluid. Because the tube is thin, the liquid plugs and vapor bubbles are formed inside it. This article presents the results of experiments using heat pipes for cooling.
\end{abstract}

Keywords: condensation, evaporation, heat, pulsating, temperature

\section{Introduction}

Recent development in semiconductor and other other mini- and micro-scale electronic technologies and continued miniaturization have led to very high increase in power density for high-performance chips. Although impressive progress has been made during the past decades, there remain serious technical challenges in thermal management and control of electronics devices or microprocessors. The two main challenges are: adequate removal of ever increasing heat flux and highly non-uniform power dissipation (Pfahl and McElroy, 2005). In fact, the micro- and power-electronics industries are facing the challenge of removing very high heat 
flux of around $300 \mathrm{~W} / \mathrm{cm}^{2}$ while maintaining the temperature below $85{ }^{\circ} \mathrm{C}$ (Agostini et al., 2007). Furthermore, due to increasing integration of devices, the power dissipation on the chip or device is getting highly non-uniform as a peak chip heat flux can be several times that of the surrounding area. Conventional cooling approaches are increasingly falling apart to deal with the high cooling demand and thermal management challenges of emerging electronic devices. Thus, highperformance chips or devices need innovative techniques, mechanisms, and coolants with high heat transfer capability to enhance the heat removal rate in order to maintain their normal operating temperature. Unless they are cooled properly, their normal performance and longevity can deteriorate faster than expected. In addition, the failure rate of electronic equipment increases with increasing operating temperature (Murshed, 2016).

\section{Cooling methods}

Engineering thermal analyses of electronic systems are based on any or all of three methods of thermal-energy transport: conduction, convection, and radiation. Conduction takes place within a medium, but without obvious transport of the medium itself. Convective heat transfer also requires a medium for energy flow, but in this instance there is mass transport of the medium. One of the most visible examples of material transport is water heated in an open kettle. The warm water rises in the center of the kettle and falls to the bottom as it becomes cooled upon transferring heat to the kettle walls. The liquid flow not only mixes the fluid, but actually aids in the rate of heat transfer in the vicinity of the walls. A very careful examination of the fluid immediately adjacent to the wall would show negligible fluid flow. In this very thin layer, heat is transferred by conduction. In a manner similar to the heated water in a kettle, circulating convective air currents in the interior of sealed electronic enclosures aid the transfer of heat energy to the cabinet wall. Upon conduction through the metal or plastic enclosure, external convective heat transfer aids removal of thermal energy from the system. Radiation heat transfer is totally unique when compared with conduction and convection in that no transport medium is required because radiation energy transport occurs via the propagation of an electromagnetic radiation field through space. Although this field typically covers the entire electromagnetic spectrum, most of the thermal radiation encountered from conventional microelectronic components and systems is located in the infrared region. All three heat transfer mechanisms obey the second law of thermodynamics in the sense that a net energy transfer flows only from a higher temperature to a lower temperature region (Elisson, 2011).

Based on heat transfer effectiveness, the existing cooling modes can be classified into four general categories which are:

- Natural convection.

- Forced convection air cooling.

- Forced convection liquid cooling. 
- Liquid evaporation.

Based on the approximate range of heat flux removal rate of these methods, it is known that liquid evaporation is the best technique followed by the forced convections of liquids and then air (Scott, 1974). However, forced air convection, which is widely used in cooling electronics such a CPU of computing devices, has very low heat removal rate (though higher than radiation and natural convection). As well known, besides heat removal mode, cooling fluids also play a major role in overall cooling performance (Pal et al. 2002).

Traditional cooling approaches, consisting typically of air-cooled heat sinks, are increasingly falling short in meeting the cooling demands of modern electronic devices with high-powered densities. These include heat pipes, thermosyphons, thermoelectric coolers and absorption refrigeration systems (24). These cooling techniques can be categorized into passive and active systems. Passive cooling systems utilize capillary or gravitational buoyancy forces to circulate the working fluid, while active cooling systems are driven by a pump or compressor for higher cooling capacity and improved performance. Heat pipes-based electronics cooling is very popular and is recently receiving great attention (Kim et al. 2008).

\section{Pulsating heat pipes}

The heat pipe is a heat transfer device that effectively utilizes evaporation and condensation to transfer heat over a long distance. A heat pipe typically consists of a container charged with a working fluid. This sealed container is divided into three sections: the evaporator, where heat is added, the adiabatic section, where no heat transfer exists, and the condenser where heat is rejected. Pulsating heat pipe is a heat transfer device that functions via thermally excited oscillating motion induced by the cyclic phase change of an encapsulated working fluid. A typical OHP consists of a train of liquid plugs and vapor bubbles which exist in serpentinearranged, interconnected capillary tubes or channels. The OHP is partially filled with a working fluid. The internal diameter of the OHP must be small enough so that liquid plugs can be separated by vapor bubbles. During operation, the continual condensation (in the condenser) and evaporation (in the evaporator) of the working fluid creates a pulsating/nonequilibrium vapor pressure field that drives fluid motion between adjacent tube/channel sections. This results in a complex flow pattern characterized by oscillatory and circulatory liquid/vapor volumes providing for both sensible (convection) and latent (phase change) heat transfer. Although the phase change heat transfer in an OHP helps to generate the oscillating motion, most of the heat is transported by sensible heat transfer. The oscillating flow and heat transfer of single phases play an important role in OHPs.

The oscillating motion in an OHP depends on the dimensions, working fluids, operating temperature, surface conditions, heat flux and total heat load, orientation, turns, and most importantly the filling ratio, $\mathrm{V}_{1} / \mathrm{V}_{\mathrm{t}}$, where $\mathrm{V}_{1}$ is the liquid volume occupied by the liquid in the 
system and $V_{t}$ is the total volume. Utilizing phase change heat transfer and oscillating convection, the heat is transferred from the evaporating section to the condensing section. Compared with a conventional heat pipe, OHP has the following unique features: an OHP is an "active" cooling device that converts heat from the heating area into the kinetic energy of liquid plugs to initiate and sustain the oscillating motion; the liquid flow does not interfere with the vapor flow because both phases flow in the same direction; the thermally driven oscillating flow inside the capillary tube effectively produces some free surfaces that significantly enhance evaporating and condensing heat transfer; the oscillating motion in the capillary tube significantly improves the forced convection in addition to the phase change heat transfer; and as the input power increases, the heat transport capability of an OHP dramatically increases (Hongbin Ma, 2015).

The basic condition for the proper functioning of the pulsating heat pipe is a value by internal diameter of the tube that has to be small enough so that the liquid slug and vapor bubble the can coexist. For these cases, the maximum internal diameter determined from the relation for the Bond number as (1):

$$
\mathrm{D}_{\text {crit }} \square 2 .
$$$$
\sqrt{\frac{\sigma \cdot G \rho_{\text {liq }} \square \rho_{\text {vap }} \curvearrowleft}{\square}}
$$

Value of Bond's number is expressed as (equation 2):

$D^{\mathrm{i}}$

$$
\frac{\text { Bo } \mathrm{l} .}{\sqrt{\frac{\sigma}{\mathrm{g}} \cdot\left[\varrho_{\text {liq }} \square \rho_{\text {vap }}[\right.}}
$$

Where $\mathrm{C}$ - surface tension $\left(\mathrm{N} \cdot \mathrm{m}^{-1}\right), \mathrm{g}-$ acceleration due to gravity $\left(\mathrm{m} . \mathrm{s}^{-2}\right), \rho_{\text {liq }}-$ density of liquid $\left(\mathrm{kg} \cdot \mathrm{m}^{-3}\right), \rho_{\text {vap }}$ - density of vapor $\left(\mathrm{kg} \cdot \mathrm{m}^{-3}\right)$.

The internal diameter of the tube is a parameter that affects the correct functioning of pulsating heat pipe. They operate optimally only in a certain range of diameters (Reay et al, 2006).

Visualization was shown to be composed of two kinds of vapor bubbles - the small circular bubbles and vapor bubbles long, so-called. Taylor's bubbles. They are formed over the whole volume of the heat pipe. The significant systems are formed, as the forces of surface tension are 
dominant. The contact angle of curvature of the vapor bubbles is dependent on the working fluid and the tube material (Fig. 1).

Figure 1: Vapor bubbles in the one meander in evaporation part of glass pulsating heat pipe

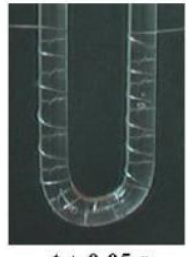

$\mathbf{t}+\mathbf{0 , 0 5} \mathrm{s}$

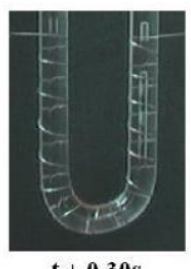

$\mathbf{t}+\mathbf{0 , 3 0 s}$

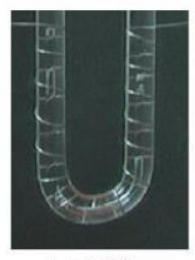

$\mathbf{t}+\mathbf{0 , 5 5 s}$
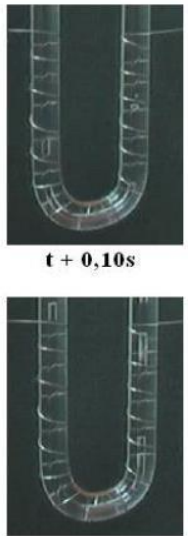

$\mathbf{t}+\mathbf{0 , 3 5 s}$

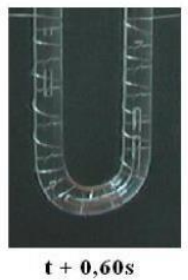

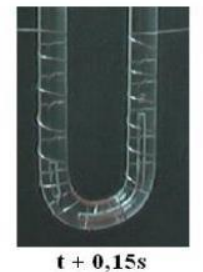

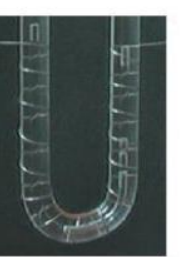

$\mathrm{t}+\mathbf{0 , 4 0 s}$

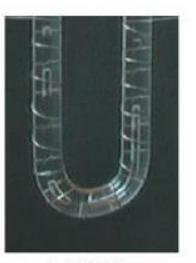

$t+0,65 s$

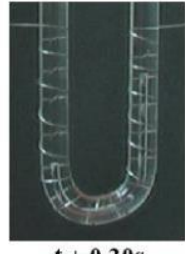

$\mathrm{t}+\mathbf{0 , 2 0 \mathrm { s }}$

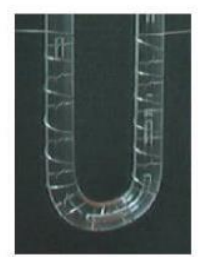

$t+0,45 s$

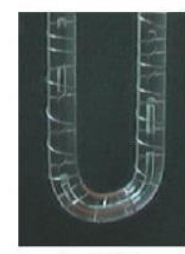

$\mathrm{t}+\mathbf{0 , 7 0 \mathrm { s }}$
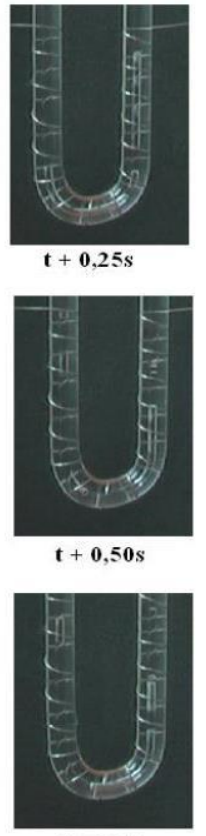

$t+0,75 s$

\section{Fluorinert FC-72 as cooling fluid}

Fluorinert FC-72 was chosen in our experiment to the physical characteristics and applicability in wide range of temperatures. Some properties at atmospheric pressure and a temperature of $20{ }^{\circ} \mathrm{C}$ are the heat capacity of $1100 \mathrm{~J} \cdot \mathrm{kg}^{-1} \cdot \mathrm{K}^{-1}$, thermal conductivity 0,057 $\mathrm{W} . \mathrm{m}^{1} . \mathrm{K}^{-1}$, surface tension $0,01 \mathrm{~N} \cdot \mathrm{m}^{-1}$, dynamic viscosity $6,4 \cdot 10^{-4} \mathrm{~Pa} . \mathrm{s}^{-1}$, density $1680 \mathrm{~kg} \cdot \mathrm{m}^{-3}$. Boiling point is $56{ }^{\circ} \mathrm{C}$ and pour point is $-90^{\circ} \mathrm{C}$, latent heat of vaporization (at normal boiling

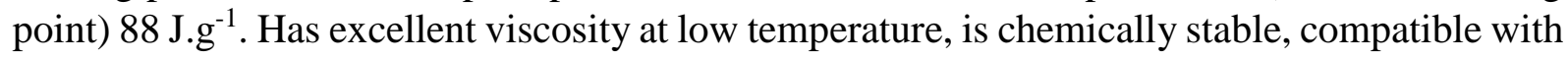
sensitive materials, including metals, plastics and elastomers, non-flammable and virtually nontoxic and does not irritate skin or eyes. Its dielectric constant is 1,75 (www.amsmaterials.com).

\section{Cooling system for electrical components}

Electrical components - bipolar transistors were used in cooling research. These components are used in railway wagons to ensure reliable and safe operation of trains and thus of rail transport. The basic requirement for the cooling system was the elimination of mechanical 
moving parts. These parts have a high probability of failure during operation. It was important to create a system that would work in both wagon operations. The first is when the wagon is in motion and the second is when the wagon is not in motion, e.g. when is in a station.

A cooling system for three components has been designed. Copper capillary with an internal diameter of 1,25 $\mathrm{mm}$ and an outside diameter of 2,45 $\mathrm{mm}$ was used for the construction. In view of the length, it is possible to create restriction 158 meanders total length of 29,07 m. For heating the evaporation section of the heat pipe was constructed evaporator with dimensions of $115 \mathrm{x}$ $200 \mathrm{~mm}$. The amount of the evaporation section was chosen to $30 \mathrm{~mm}$. To ensure even heating and the shape of the heat pipe is rolled into the evaporator and this part is filled with a mixture of epoxy resin and copper powder to ensure the thermal conductivity. Temperature in the evaporator was scanned. Two thermocouples NiCr-Ni were placed into the mixture. Adiabatic section was isolated from the influence of the external environment and the influence of the temperature of the evaporator. In the next phase it was necessary to fill the heat pipe working fluid Fluorinert FC- 72. The value of the inner diameter of the copper capillary satisfies the critical value of the internal diameter. According to the results on the effect of filling ratio of the thermal performance was heat pipe filled to $50 \%$ of its capacity (Fig.2).

Figure 2: 158 meanders pulsating heat pipes and electrical components for cooling
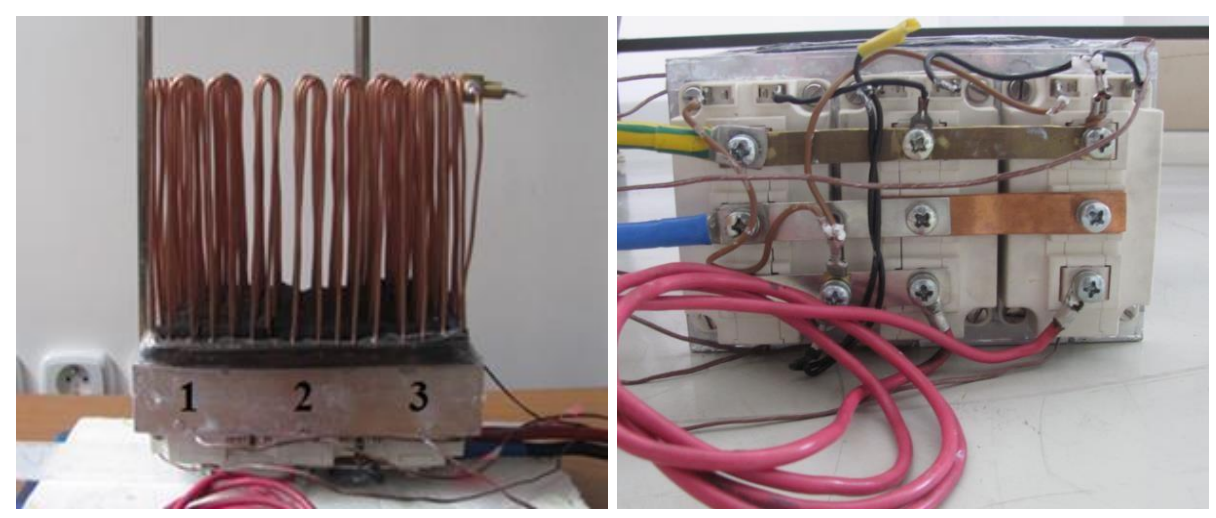

To investigate the contact temperature of the electric energy converters for different values the supplied heat was compiled experimental device according. Thermal performance of the closed loop pulsating heat pipe was measured at different air velocity (Fig. 3). Various air velocity was secured by regulating fan speed. Air enters the ventilator through a fixer pipe of length $1 \mathrm{~m}$, at the beginning of the anemometer was installed. Anemometer was measured velocity of inlet air, whereby the calculated mass flow rate of air. 


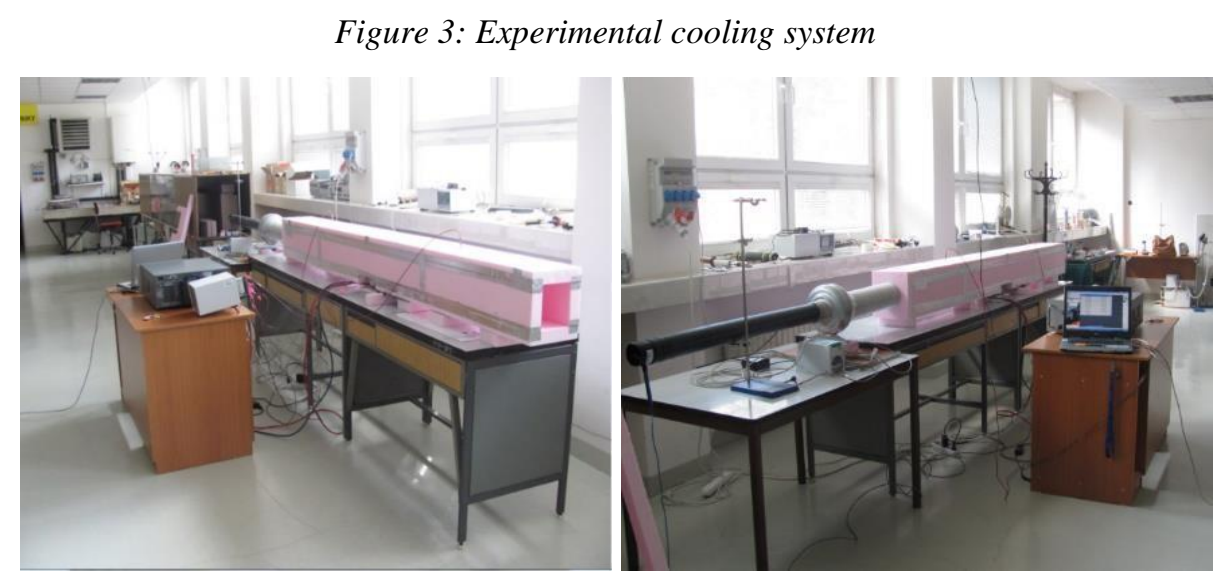

The length of the tunnel in front of the tube and back of the tube was determined from the hydraulic diameter of the tunnel. Pulsating heat pipe was placed in the middle of the tunnel. For sensing the temperature in front of the tube and back the tube was used for paired Pt100 resistance sensors Evaporation section was warmed by heat dissipation in electronic devices connected to the evaporator. Electric energy converters were connected to a source of direct current. All devices were connected to the input of logger AHLBORN ALMEMO 5690-2.

\section{Results}

In cooling of electric energy converters by means of closed loop pulsating heat pipe was necessary to satisfy the condition that the temperature of the contact areas of electric energy converters does not exceed $100{ }^{\circ} \mathrm{C}$, which can result in damage to these components. Air velocities were selected from 2 to $16 \mathrm{~m} / \mathrm{s}$ according to the operating conditions of the wagons. The component load has been selected for performance 50,100 and $150 \mathrm{~W}$ for each component. Measurements were performed in the laboratory conditions. In Figures 4, 5 and 6 show the results of the measurement. 


\title{
$2^{\text {nd }}$ International Conference on Research in SCIENCE ENGINEERING AND TECHNOLOGY
}

22-24 November, 2019

\author{
Paris, France
}

Figure 4: Cooling system for load $50 \mathrm{~W}$

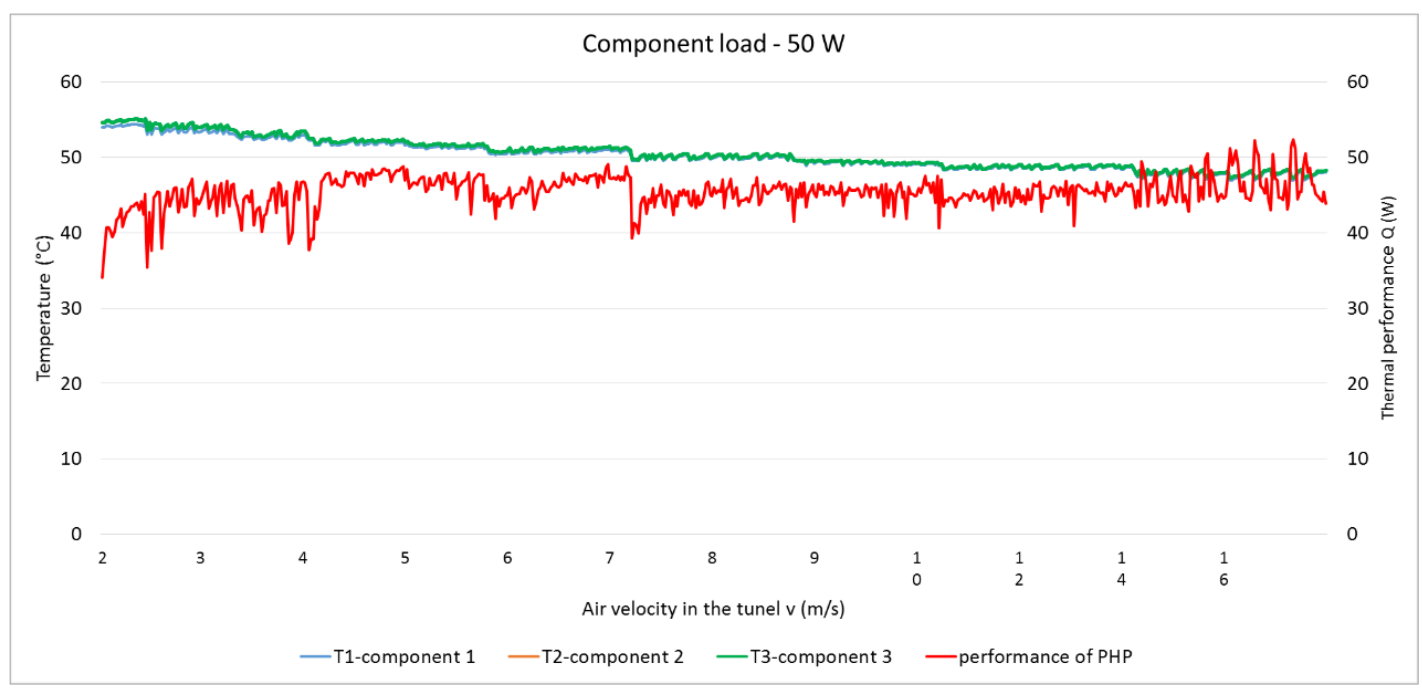

Figure 5: Cooling system for load $100 \mathrm{~W}$

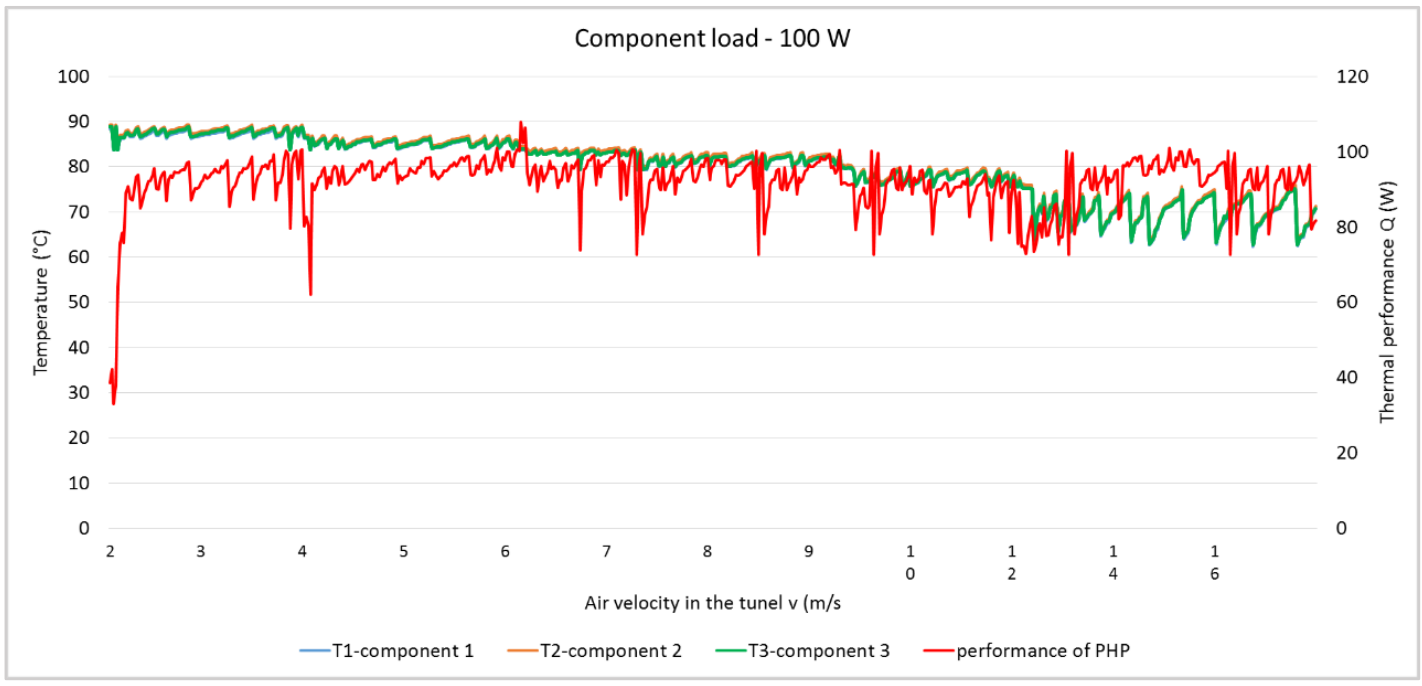

Figure 6: Cooling system for load $150 \mathrm{~W}$ 


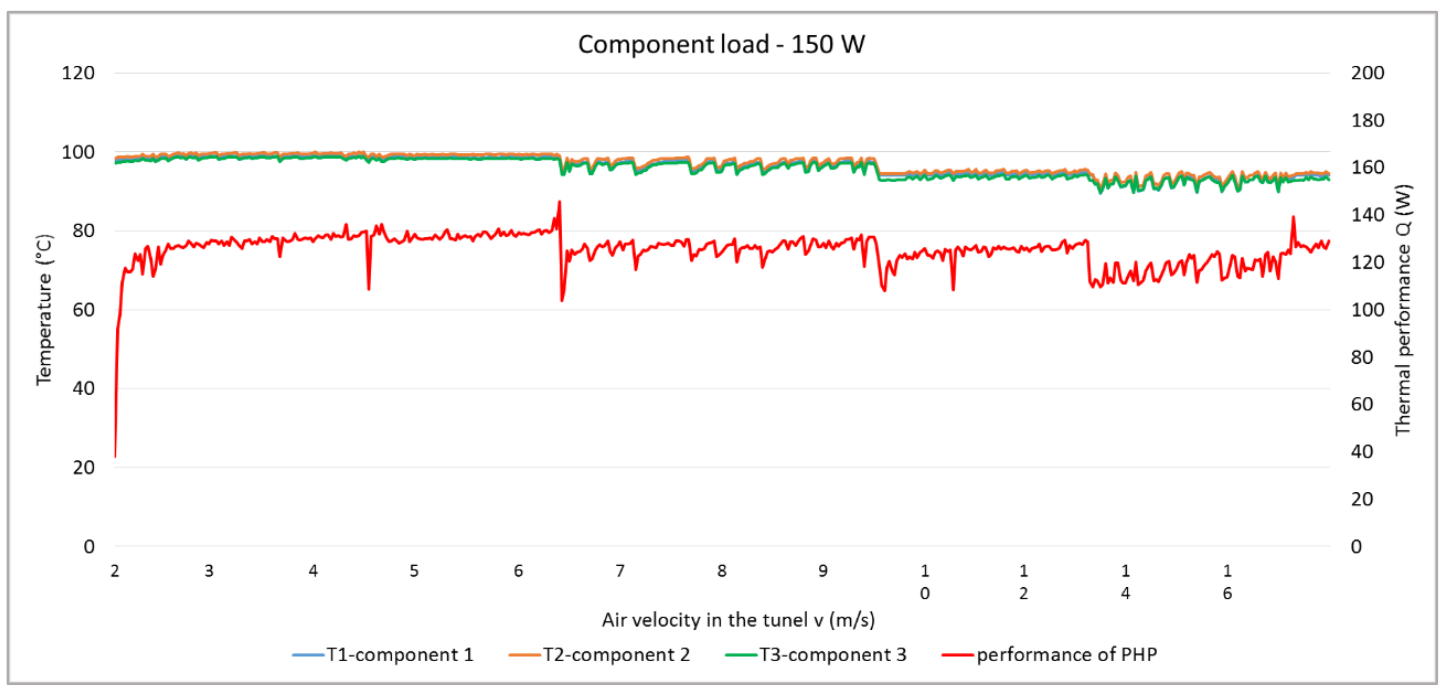

\section{Conclusion}

Our goal is to create an efficient and effective cooling system. An important requirement is the removal of mechanical moving parts, which often cause failure. Pulsating heat pipes are a part to research in this area. Special type - closed loop pulsating heat pipe were used in our case. At first we examined the filling ratio of closed loop pulsating heat pipe. We chose Fluorinert FC-72 as working fluid. Fluorinert does not destroy components in the event of a failure.To search the effectiveness of the cooling system was created wind tunnel. In the wind tunnel we measured the temperature of electronic components and thermal performance closed loop pulsating heat pipe. Value of transferred thermal performances of closed loop pulsating heat pipe with 158 meanders were expressed using a colorimetric equation. Operation of heat pipes is provided by phase changes - evaporation and condensation. The results of the experiment show that the cooling of electrical components with a load of $50 \mathrm{~W}$ and $100 \mathrm{~W}$ is effective for all air velocities. Gradually increasing the power up to $150 \mathrm{~W}$ and the reduction rate of $6 \mathrm{~m} / \mathrm{s}$ we reached $100{ }^{\circ} \mathrm{C}$, which we have also completed the measurements. Results show that cooling systems with non-conventional type of heat pipes has potential in electrical application. But it should be defined in detail the value of the loss of heat to be lead away from these systems. The aim is to create a system, especially under the natural convection and meet this objective can be achieved through a system of loop heat pipe. If the technical applications and limited space is provided by forced convection, it is interesting to use the system with the pulsating heat pipe. Our next aim is to research and proposal cooling systems for higher values of thermal load. Solution to this problem will undertake a research cooling system with pulsating heat pipe under the natural convection and increased heat dissipation in the loop heat pipe. 


\section{Acknowledgment}

This work was supported under the project of Operational Programme Research and Innovation: Research and development activities of the University of Zilina in the Industry of 21st century in the field of materials and nanotechnologies, No. 313011T426. The project is cofunding by European Regional Development Fund.

\section{References}

[1] Agostini B, Fabbri M, Park JE, Wojtan L, Thome JR and Michel B., (2007). State of the art of high heat flux cooling technologies. Heat Transfer Engineering. 28:258-281. Available: https://doi.org/10.1080/01457630601117799.

[2] Elison G.N. (2010). Thermal Computations for Electronics. Published by CRC Press, Taylor \& Francis Group. ISBN-13: 978-1-4398-5076-3, pp. 418.

[3] Florková, Z., Šedivý, Š., Pepucha, L'. (2017) Analysis of results of the aggregate microtexture evaluation by volumetric characteristics. MATEC Web of Conference, Published by EDP Sciences, Vol. 117, Number UNSP 00046, pp. 8.

[4] Dudak, J., Gašpar, G., Šedivý, Š., Pepucha, L., Florková, Z. (2017) Road structural elements temperature trends diagnostics using sensory system of own design. IOP Conference SeriesMaterials Science and Engineering, published by IOP Publishing LTD., Volume 236, pp.

9.

[5] Hongbin Ma (2015). Oscillating heat pipes. Springer. ISBN-13: 978-1493925032, pp. 427 ,

[6] Kim YJ, Joshi YK and Fedorov AG. An absorption miniature heat pump system for electronics cooling. International Journal of Refrigeration. 2008; 31(1):23-33.

[7] Murshed, S.M.S. (2016). Electronics Cooling. Published by ExLi4EvA. ISBN-13: 97895351-2406-1, pp. 180.

[8] Pal A, Joshi YK, Beitelmal MH, Patel CD and Wenger TM. (2002). Design and performance evaluation of a compact thermosyphon. IEEE Transactions on Components and Packaging Technologies. 25(4): 601-607.

[9] Pfahl RC and McElroy J. The 2004 international electronics manufacturing initiative (iNEMI) technology roadmaps. Proceedings of the IEEE. 2005; 2005:1-7.

[10] Reay, D. and Kew, P. (2006). Heat Pipes Theory, Desing and Applications. UK: Fifth Edition, ISBN-13: 978-0-7506-6754-8. 


\section{$2^{\text {nd }}$ International Conference on Research in SCIENCE ENGINEERING AND TECHNOLOGY}

22-24 November, $2019 \quad$ Paris, France

[11] Sott WA. (1974). Cooling of Electronic Equipment. New York: John Wiley and Sons.

[12] http://www.amsmaterials.com/products/Fluorinert-FC\%252d72.html. 